\title{
Influencia de los parámetros de soldeo fuerte en la microestructura y propiedades mecánicas de la unión de la aleación base níquel Hastelloy B2
}

\author{
José Carlos Sotelo ${ }^{\mathrm{a}, \bowtie}$, Marcos González ${ }^{\mathrm{a}}$, Enrique Porto ${ }^{\mathrm{b}}$ \\ ${ }^{a}$ Centro Tecnológico AIMEN, Planta de Tecnologías de Unión, Relva 27A, 36410, O Porriño, Pontevedra, España \\ ${ }^{b}$ Departamento de los Materiales e Ingeniería Metalúrgica. Escuela Técnica Superior de \\ Ingenieros Industriales. Universidad de Vigo, Pontevedra, España \\ Autor para la correspondencia: jsotelo@aimen.es
}

Enviado: 14 Junio 2013; Aceptado: 8 Febrero 2014; Publicado on line: 29 August 2014

\begin{abstract}
RESUMEN: Se realizó un estudio pormenorizado del proceso de soldeo fuerte en horno de alto vacío de la aleación base níquel Hastelloy B2 fortalecida por solución sólida. En una primera fase del trabajo se seleccionó el material de aporte acorde al material objeto de unión y a la fuente de calentamiento seleccionada. Posteriormente, se evaluó la influencia del gap (50 y 200 micrómetros) y tiempo de permanencia a temperatura de soldeo (10 y 90 minutos) sobre los microconstituyentes de la unión, relacionando la microestructura con las propiedades mecánicas de la junta. Los análisis metalográficos mostraron una unión constituida por una matriz rica en níquel, siliciuros de níquel y compuestos ternarios. Finalmente, los resultados de los ensayos mecánicos a esfuerzos cortantes mostraron una elevada resistencia para gaps de 50 micrómetros y tiempos de permanencia de 90 minutos.
\end{abstract}

PALABRAS CLAVE: Hastelloy; Níquel; Soldadura fuerte; Vacío

Citation / Cómo citar este artículo: Sotelo, J.C., González, M., Porto, E. (2014) "Influencia de los parámetros de soldeo fuerte en la microestructura y propiedades mecánicas de la unión de la aleación base níquel Hastelloy B2". Rev. Metal. 50(3): e019. doi: http://dx.doi.org/10.3989/revmetalm.019.

\begin{abstract}
Influence of the brazing parameters on microstructure and mechanical properties of brazed joints of Hastelloy B2 nickel base alloy. A study of the high vacuum brazing process of solid solution strengthened Hastelloy B2 nickel alloy has been done. A first stage of research has focused on the selection of the most appropriate brazing filler metal to the base material and vacuum furnace brazing process. The influence of welding parameters on joint microstructure constituents, relating the microstructure of the joint to its mechanical properties, has been evaluated. Two gaps of 50 and 200 micrometers, and two dwell times at brazing temperature of 10 and 90 minutes were studied. The braze joint mainly consists of the nickel rich matrix, nickel silicide and ternary compounds. Finally, the results of this study have shown the high bond strength for small gaps and increased dwell times of 90 minutes.
\end{abstract}

KEYWORDS: Hastelloy; Nickel; Brazing; Vacuum

Copyright: (C) 2014 CSIC. This is an open-access article distributed under the terms of the Creative Commons Attribution-Non Commercial (by-nc) Spain 3.0 License.

\section{INTRODUCCIÓN}

La industria petroquímica se caracteriza por trabajar en medios altamente corrosivos como por ejemplo en atmósferas con elevados contenidos en ácido clorhídrico, sulfúrico o acético. Los altos costes económicos que provocan los efectos de la corrosión en tuberías o recipientes, motivaron el desarrollo de aleaciones con alta resistencia a estos medios. De esta necesidad nacieron las aleaciones de níquel Hastelloy 
B2 cuya composición química se caracteriza por su elevado contenido en molibdeno. Este elemento le confiere una excelente resistencia a la corrosión, elevada estabilidad térmica y baja dilatación hasta temperaturas próximas a $1100{ }^{\circ} \mathrm{C}$, (Davis, 2000; Cao et al., 1994).

El empleo de las aleaciones base níquel hace que en la mayoría de los casos se requiera que éstas sean fácilmente soldables. Las operaciones de soldeo adquieren verdadera importancia, especialmente en la fabricación de componentes de altas prestaciones para el sector aeronáutico y la industria petroquímica (Rodríguez et al., 1998).

Dentro de las diferentes tecnologías de unión que se emplean habitualmente en la soldadura de estas aleaciones, se encuentra el proceso de soldeo fuerte. Esta tecnología es ampliamente utilizada en diferentes sectores industriales como automoción, aeronáutica o electrónica. Las ventajas que ofrece el soldeo fuerte para la unión de aleaciones de níquel frente a los procesos de soldadura por arco eléctrico son múltiples, destacando de entre todas ellas las reducidas temperaturas del proceso, la posibilidad de unir aleaciones de níquel complejas, como son las aleaciones fortalecidas por solución sólida o por dispersión de óxidos (OSD) y ser un proceso fácilmente automatizable y de elevada productividad (Roberts, 2003; Schwartz, 2003).

Cualquier operación de soldadura debe ser cuidadosamente ajustada en lo relativo al aporte térmico, dada su gran influencia en las propiedades finales de la unión soldada. En este sentido, la composición química de la aleación de níquel junto a los parámetros de soldeo ejercen un efecto muy definido en la soldabilidad, y el conocimiento de esta influencia es de suma importancia a la hora de definir los procedimientos de soldeo.

En la actualidad se desconocen los efectos del ciclo térmico de soldeo fuerte de aleaciones Hastelloy B2 en las propiedades mecánicas de la unión, por ello, el presente trabajo pretende estudiar los efectos que ejercen en la calidad de la unión, el tiempo de permanencia a temperatura de soldeo $\left(\mathrm{t}_{\mathrm{Ts}}\right)$, la geometría de unión (gap) y el material de aporte.

\section{MATERIALES Y MÉTODOS}

\subsection{Selección del material de aporte}

El soldeo fuerte de la aleación Hastelloy B2, cuya composición química se muestra en la Tabla 1, requiere la selección de materiales de aporte. Éstos,

TABLA 1. Composición química de la aleación Hastelloy B-2 ( $\%$ en peso)

\begin{tabular}{lccccccccc}
\hline Ni & $\mathbf{C o}$ & $\mathbf{C r}$ & $\mathbf{M o}$ & $\mathbf{F e}$ & $\mathbf{S i}$ & $\mathbf{M n}$ & $\mathbf{C}$ & $\mathbf{P}$ & $\mathbf{S}$ \\
\hline Resto & 1,00 & 1,00 & 28,00 & 2,00 & 0,10 & 1,00 & 0,02 & 0,02 & 0,01 \\
\hline
\end{tabular}

TABLA 2. Composición química del material de aporte Ni650 ( $\%$ en peso)

\begin{tabular}{lccccccc}
\hline Ni & $\mathbf{C}$ & $\mathbf{B}$ & $\mathbf{S i}$ & $\mathbf{P}$ & $\mathbf{C r}$ & $\mathbf{F e}$ & $\mathbf{C o}$ \\
\hline Resto & $<0,06$ & $<0,02$ & 9,80 & $<0,02$ & 18,70 & 2,84 & 0,10 \\
\hline
\end{tabular}

deben de presentar buenas propiedades tanto mecánicas como de resistencia a la corrosión y una buena afinidad metalúrgica con el material base objeto de unión. Tales requisitos obligan a la utilización de aportes de la familia de aleaciones base níquel según UNE-EN ISO 17672 (2010). De las diferentes composiciones que se recogen en la norma, se seleccionó la aleación Ni650 dadas las buenas propiedades mecánicas que confiere a la unión (Grushko et al., 1984). La Tabla 2 muestra la composición química a la aleación Ni650 seleccionada.

Para determinar la temperatura de soldeo, se realizó un estudio mediante análisis térmico diferencial (ATD) del material de aporte utilizado. Para ello, se utilizó un analizador térmico diferencial Setaram modelo Setsys Evolution 16/18. La velocidad de calentamiento isotérmico fue de $10^{\circ} \mathrm{C} \mathrm{min}{ }^{-1}$, utilizando una atmósfera inerte de argón con un flujo de $70 \mathrm{ml} \mathrm{min}{ }^{-1}$. Se utilizaron crisoles de alúmina en los que se depositaron alrededor de $25 \mathrm{mg}$ de la aleación Ni650.

\subsection{Preparación de las probetas de ensayo}

Para estudiar los efectos que tienen sobre la bondad de la unión los diferentes parámetros que intervienen en el proceso y relacionar éstos con la geometría de la unión, se efectuaron pruebas de soldeo fuerte combinando dos aberturas de unión $(50$ y $200 \mu \mathrm{m})$ y dos tiempos a temperatura de soldeo (10 y 90 minutos).

Las probetas de soldeo se componen de dos chapas de $100 \mathrm{~mm}$ de longitud, $40 \mathrm{~mm}$ de ancho y $1 \mathrm{~mm}$ de espesor. Para asegurar aberturas de 50 y $200 \mu \mathrm{m}$, se emplearon galgas calibradas punteando las probetas mediante puntos de soldadura TIG (Toungsten Inert Gas) por ambos extremos, dejando un solape de $4 \mathrm{~mm}$. Los puntos de soldadura se realizaron con un equipo TIG-AC/DC Fronius modelo Magic-Wave 3000.

Tras la soldadura fuerte en horno de alto vacío, los insertos y puntos TIG ubicados en los extremos de las probetas fueron mecanizados con el objetivo de no alterar los posteriores ensayos mecánicos a los que se vieron sometidas.

\subsection{Ciclo térmico de soldeo}

Los ciclos térmicos de soldeo se llevaron a cabo en un horno de alto vacío TAV modelo TAV333. La definición del ciclo térmico, se realizó teniendo en 
cuenta la naturaleza de la aleación objeto de unión, que presenta una estructura monofásica cúbica centrada en las caras (fase $\alpha$ ) a una temperatura superior a los $870{ }^{\circ} \mathrm{C}$. La fase $\alpha$ puede ser retenida si se produce un enfriamiento rápido a partir de $870^{\circ} \mathrm{C}$ hasta una temperatura inferior a $600{ }^{\circ} \mathrm{C}$ (Brooks et al., 1989). Este intervalo de temperaturas se ha tenido en cuenta en el ciclo térmico de soldeo, ya que si la velocidad de enfriamiento no es la suficiente, o se mantiene la aleación a una temperatura dentro de ese intervalo crítico de temperaturas, se produce la precipitación del compuesto $\mathrm{Ni}_{4} \mathrm{Mo}$. La formación de este intermetálico ejerce un efecto de fragilización, incidiendo negativamente en las propiedades mecánicas de la aleación (Brooks et al., 1990).

\subsection{Caracterización de las uniones}

Cada unión soldada se caracterizó microestructural y mecánicamente. El análisis microestructural del material de aporte, material base y uniones realizadas, se efectuó mediante microscopía óptica utilizando un banco metalográfico Leica modelo MEF 4 dotado de un sistema electrónico de adquisición de imágenes. Como método complementario, se empleó un microscopio electrónico de barrido de emisión de campo (FE-SEM) de ultra-alta resolución Hitachi S-4800 II. El equipo dispone de un sistema de microanálisis por espectroscopía de dispersión de energía de rayos X (EDS) de Röntec. Finalmente y para identificar las diferentes fases y compuestos que se presentan en las uniones realizadas, se utilizó un detector de electrones retrodispersados (EBSD) HKL Technology de Oxford Instruments.

La caracterización mecánica se realizó mediante un ensayo de cizalla sobre tres probetas por cada ciclo térmico. Asimismo, las probetas sometidas a este ensayo permitieron controlar, por inspección visual, la calidad de la unión (penetración, porosidad, etc.), información que facilitó el análisis comparativo entre las distintas condiciones de soldeo.

Para la caracterización mecánica de las uniones se utilizó una máquina universal de ensayos de $250 \mathrm{kN}$ de capacidad de MTS, modelo 81022. Al objeto de determinar las durezas de las fases formadas en la unión y de esa forma indirecta, caracterizar la ductilidad de la misma, se empleó un microdurómetro Vickers Shimadzu modelo HMV-2T con una fuerza aplicada de HV 0,01 .

\section{RESULTADOS Y DISCUSIÓN}

\subsection{Análisis térmico diferencial}

En el diagrama obtenido en el análisis térmico diferencial de la aleación Ni650 (no se muestra en este trabajo), únicamente aparecen los picos correspondientes a la reacción endotérmica de fusión a $1138,7{ }^{\circ} \mathrm{C}$ seguido del pico exotérmico a $1124,5{ }^{\circ} \mathrm{C}$ correspondiente a la solidificación, por lo que se supone temperatura de fusión única. Este resultado indica que el material de aporte tiene composición eutéctica o muy próxima a ella.

\subsection{Análisis microestructural}

El análisis por microscopía óptica muestra la evolución microestructural de las diferentes uniones realizadas con el aporte Ni650 a diferentes aberturas y tiempos a temperatura de soldeo. La microestructura del cordón de soldadura con gap de $200 \mu \mathrm{m}$ muestra tres regiones claramente diferenciadas: interfase de unión, zona de granos columnares y la región central del cordón (Fig. 1).

Los valores de la microdurezas muestran un incremento de la dureza en la interfase de unión y, especialmente, en el centro del cordón de soldadura, posiblemente por la presencia de compuestos intermetálicos de elevada dureza. Las microdurezas en la unión [gap 200, $\mathrm{t}_{\mathrm{Ts}}$ 90] no presentan diferencias apreciables con respecto a la unión con $\mathrm{t}_{\mathrm{Ts}} 10$. En ambas muestras se produce una disminución de la dureza en la estructura columnar, con valores similares a los del material base.

En las uniones configuradas con un diseño de gap 50 hay presencia de la región central eutéctica en las probetas con $\mathrm{t}_{\mathrm{Ts}} 10$, no así en la unión con $t_{T s} 90$ tal y como se aprecia en las micrografías de la Figura 2. Asimismo, la región de la interfase de unión presentó menor espesor en ambas probetas.
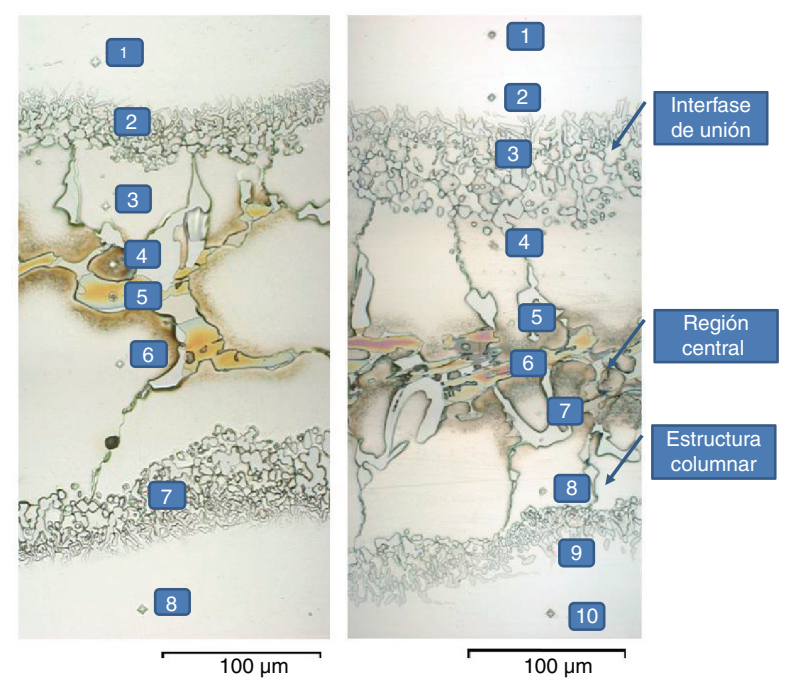

Figura 1. Microestructura de las uniones con gap de $200 \mu \mathrm{m}$ en las que se muestran las huellas del ensayo de microdureza. Izquierda: unión con $\mathrm{t}_{\mathrm{Ts}} 10$, huellas 1 y 8 correspondientes al material base. Derecha: unión con $\mathrm{t}_{\mathrm{Ts}} 90$, huellas 1 y 10 correspondientes al material base. 

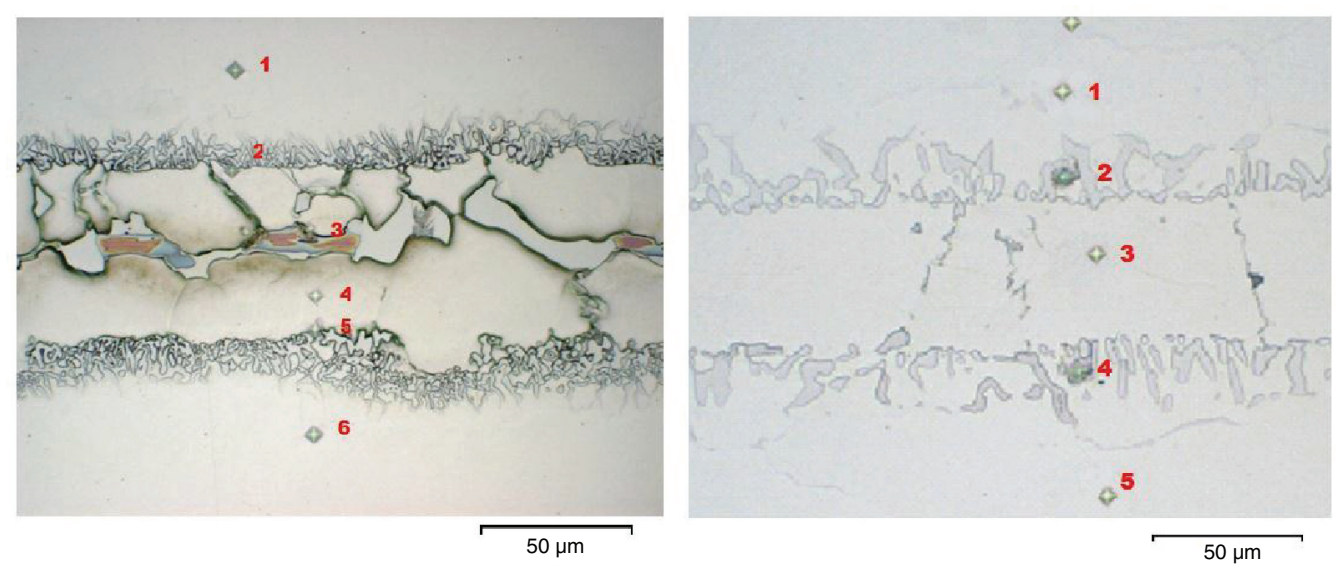

Figura 2. Resultados del ensayo de microdureza de las uniones con gap de $50 \mu \mathrm{m}$. Izquierda: unión con $\mathrm{t}_{\mathrm{Ts}} 10$. Derecha: unión con $\mathrm{t}_{\mathrm{Ts}} 90$.

Los valores de microdurezas de la unión [gap $\left.50, t_{\mathrm{Ts}} 10\right]$ muestran un incremento de la dureza en la interfase de unión y, especialmente, en la región central del cordón de soldadura, alcanzándose valores superiores a $2000 \mathrm{HV} 0,01$, en la cual se produce una concentración de compuestos intermetálicos de elevada dureza. Por otra parte, los valores de la microdurezas en la unión [gap 50, $\mathrm{t}_{\mathrm{Ts}}$ 90] revelaron un endurecimiento en la interfase de unión, resultado muy similar al obtenido con $\mathrm{t}_{\mathrm{Ts}} 10$. En la región central, se produjo una caída de la dureza, con valores de dureza similares a los del material base.

En la Figura 3 se muestra una microfotografía SEM de la región de la unión con un gap de $200 \mu \mathrm{m}$ $\mathrm{y}_{\mathrm{Ts}} 10$. La composición química obtenida mediante EDS en diferentes zonas de la muestra se recoge en la Tabla 3. Se observan fases ricas en molibdeno con presencia de silicio y en menor cuantía níquel. Estas fases se pueden clasificar en función de su morfología, apareciendo fases de gran tamaño en el interior del cordón de soldadura y precipitados finos en la interfase de unión. Finalmente, también se evidenciaron zonas ricas en níquel y silicio con escasa o nula presencia de molibdeno y cromo.

Estas fases, se identificaron mediante análisis por difracción de electrones retro-dispersados (EBSD) en los mismos puntos en los que se obtuvieron los resultados por EDS (Fig. 3). Se identificó la presencia de compuestos ternarios MoNiSi, intermetálicos $\mathrm{Ni}_{3} \mathrm{Si}$ y la fase $\gamma$ (Kim et al., 2007), como se muestra en la Tabla 4.

Del mismo modo se realizaron análisis químicos mediante EDS (Tabla 5) y EBSD en diferentes regiones del cordón de soldadura de la unión con gap 200 y t $_{\mathrm{Ts}} 90$ (Fig.4). Se observa, que un aumento del tiempo a temperatura de soldeo disminuye la región central eutéctica, en donde se concentran los elementos depresores del punto de fusión de la

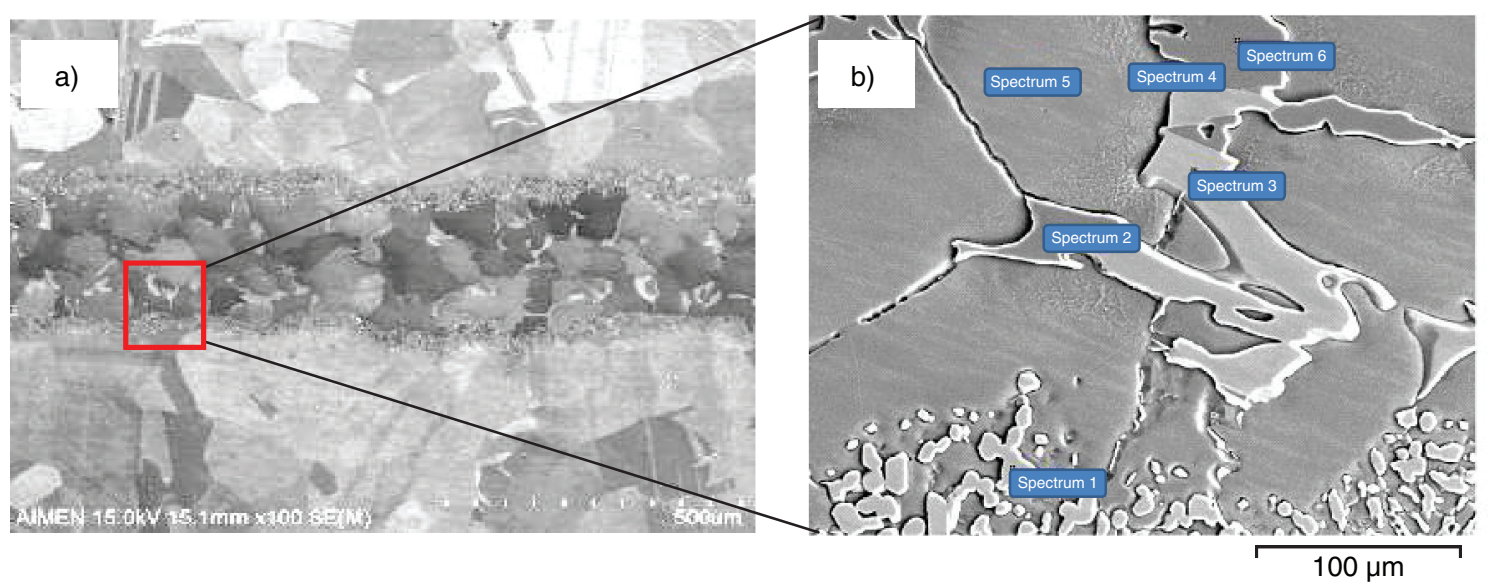

FIGURA 3. a) Imagen SEM de la probeta con gap $200 \mathrm{y} \mathrm{t}_{\mathrm{Ts}} 10$; b) Imagen SEM de la zona enmarcada en el recuadro de la figura a). 
TABla 3. Composición química, expresada en $\%$ peso, obtenida mediante EDS en diferentes zonas de la imagen SEM que aparece en la Figura 3

\begin{tabular}{lrrcccc}
\hline Espectros & Si & Cr & Mn & Fe & Ni & Mo \\
\hline 1 & 9,07 & 4,44 & - & 0,28 & 40,25 & 45,95 \\
2 & 19,68 & 2,21 & - & - & 77,58 & - \\
3 & 9,53 & 4,84 & - & - & 40,20 & 45,43 \\
4 & 7,39 & 12,97 & 0,51 & 0,73 & 74,69 & 3,72 \\
5 & 5,24 & 14,42 & 0,38 & 0,69 & 73,05 & 6,22 \\
6 & 19,91 & 2,06 & 0,25 & - & 77,42 & - \\
\hline
\end{tabular}

TABLA 4. Fases identificadas mediante EBSD en las diferentes zonas que aparecen en la Figura 3

\begin{tabular}{llll}
\hline Espectro & $\begin{array}{c}\text { Longitud celda } \\
\text { unitaria (̊) }\end{array}$ & $\begin{array}{c}\text { Ángulo celda } \\
\text { unitaria }\left({ }^{\circ}\right)\end{array}$ & Fase \\
\hline 1 y 3 & 4,$75 ; 4,75 ; 7,58$ & $90,90,120$ & MoNiSi \\
2 y 6 & 3,$51 ; 3,51 ; 3,51$ & $90,90,90$ & $\mathrm{Ni}_{3} \mathrm{Si}$ \\
4 y 5 & 3,$52 ; 3,52 ; 3,52$ & $90,90,90$ & $\mathrm{Ni}$ \\
\hline
\end{tabular}

aleación Ni650. Los resultados obtenidos muestran la presencia de compuestos ternarios MoNiSi, intermetálicos $\mathrm{Ni}_{3} \mathrm{Si}$ y la fase $\gamma$.

\subsection{Ensayo a cizalla}

En la Tabla 6 se recogen los resultados obtenidos en el ensayo de cizalla de las probetas soldadas a diferentes gaps y $\mathrm{t}_{\mathrm{Ts}}$. En todas las muestras se

Tabla 5. Composición química, expresada en \% peso, obtenida mediante EDS en diferentes zonas de la imagen SEM que aparece en las Figura 4

\begin{tabular}{lcrcccc}
\hline \multicolumn{7}{c}{ Espectros zona 1 } \\
\hline Espectro & $\mathbf{S i}$ & $\mathbf{C r}$ & $\mathbf{M n}$ & $\mathbf{F e}$ & $\mathbf{N i}$ & $\mathbf{M o}$ \\
\hline 1 & 10,07 & 2,61 & - & - & 85,67 & - \\
2 & 7,34 & 2,04 & - & - & 89,35 & - \\
3 & 4,13 & 13,34 & 0,48 & 0,97 & 78,52 & 2,56 \\
4 & 7,10 & 4,96 & - & - & 46,40 & 41,54 \\
5 & 9,77 & 2,15 & - & - & 87,49 & - \\
6 & 4,74 & 11,12 & 0,46 & 1,10 & 79,17 & 3,42 \\
\hline \multicolumn{7}{c}{ Espectros zona 2 } \\
\hline Espectro & $\mathbf{S i}$ & $\mathbf{C r}$ & $\mathbf{M n}$ & $\mathbf{F e}$ & $\mathbf{N i}$ & $\mathbf{M o}$ \\
\hline 1 & 6,00 & 3,70 & 0,18 & 0,32 & 46,73 & 43,08 \\
2 & 2,75 & 11,46 & 0,26 & 1,12 & 79,02 & 5,39 \\
3 & 6,35 & 3,91 & 0,07 & - & 46,91 & 42,76 \\
4 & 3,26 & 12,05 & 0,27 & 1,11 & 78,63 & 4,69 \\
5 & 2,64 & 12,05 & 0,33 & 1,18 & 79,05 & 4,75 \\
6 & 5,85 & 3,45 & 0,07 & 0,38 & 48,01 & 42,24 \\
\hline
\end{tabular}

produce rotura por el material de aporte siendo la unión con gap 50 y t$_{\mathrm{Ts}} 90$ la que presentó una mayor carga de rotura. Tras el ensayo, se realizó una inspección visual de la superficie de fractura de todas las probetas ensayadas. En ellas se observó una buena capacidad de propagación y mojado del material de aporte a excepción de la muestra con gap 200 y $\mathrm{t}_{\mathrm{Ts}}$ 10 , la cual mostró zonas de la junta con faltas de llenado.

\subsection{Análisis fractográfico}

La superficie de fractura de la unión con gap 200 y $\mathrm{t}_{\mathrm{Ts}} 10$, mostró regiones de la superficie sin cubrir por el aporte a causa de una clara falta de capilaridad del mismo (Fig. 5a). La superficie de unión mostró una textura facetada con un elevado tamaño de los compuestos intermetálicos presentes en la unión (Fig. 5b). El análisis químico mediante EDS reveló que eran compuestos del tipo MoNiSi.

Finalmente, el estudio mediante microscopía óptica de la sección de la superficie de fractura reveló la dirección de la propagación de las grietas, dirección normal a los esfuerzos de cortadura provocados en el ensayo. Se evidenció una fractura de carácter frágil con presencia de grietas y microgrietas cuya propagación transcurre, fundamentalmente, a través de los compuestos intermetálicos por un mecanismo de descohesión intercristalina.

\section{CONCLUSIONES}

La microestructura de la unión con aporte Ni650 y gap 50 muestra para $t_{T s} 10$, tres regiones claramente diferenciadas: región central eutéctica en la que se han identificado compuestos del tipo MoNiSi y $\mathrm{Ni}_{3} \mathrm{Si}$, así como cristales primarios de fase $\gamma$, zona de de estructura columnar de fase $\gamma$ e interfase de unión. Esta estructura se ve modificada por el incremento del $\mathrm{t}_{\mathrm{Ts}}$ a 90 minutos, transformando la región central eutéctica y zona de granos columnares a una estructura de fase $\gamma$.

Los efectos del incremento del $\mathrm{t}_{\mathrm{T}} \mathrm{S}$ sobre la microestructura de la unión con aporte Ni650 y gap 200 se traducen en un incremento del espesor de la zona de granos columnares y reducción de la región central eutéctica, en la que se identificaron los mismos microconstituyentes presentes en las uniones con gap 50 (MoNiSi, $\mathrm{Ni}_{3} \mathrm{Si}$ y fase $\gamma$ ).

La técnica de ensayo por EBSD ha demostrado ser una herramienta de relevante utilidad en la caracterización microestructural de las distintas fases que componen una unión por soldeo fuerte. En este sentido, cabe destacar la complejidad a la hora de caracterizar determinadas fases y compuestos intermetálicos que se generan en la unión por microscopía electrónica, EDS o mediante la técnica convencional de difracción de rayos $\mathrm{X}$. 


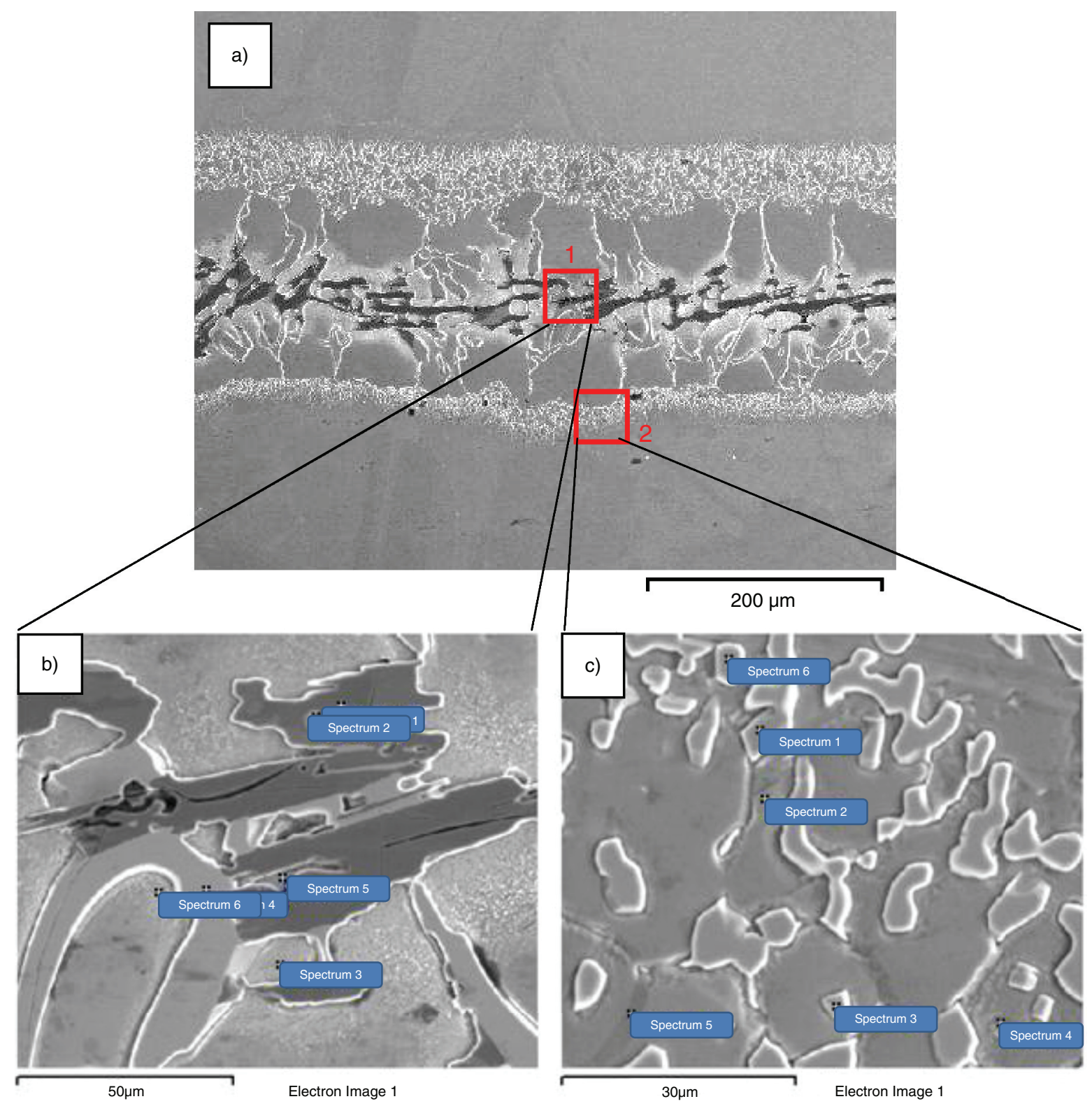

FIgURA 4. a) Imagen SEM de la unión con gap $200 \mathrm{y} \mathrm{t}_{\mathrm{Ts}} 90$ en la que señalan las zonas 1 y 2; b) Imagen SEM de la zona 1 y c) Imagen SEM de la zona 2.

El estudio fractográfico reveló un micromecanismo de fractura frágil por descohesión intercristalina en todas las uniones a consecuencia de las formación de compuestos intermetálicos frágiles y duros. Este mecanismo de fractura está presente incluso en aquellas uniones que han permanecido prolongados tiempos a temperatura de soldeo a fin de difundir aquellos elementos formadores de los compuestos intermetálicos en el material base.

Los ensayos mecánicos practicados en las uniones con aporte Ni650 mostraron rotura por el aporte en todas las probetas ensayadas. La observación visual de la superficie de fractura reveló faltas de capilaridad principalmente en las uniones con gap 200. El incremento del $\mathrm{t}_{\mathrm{Ts}}$ mejoró la resistencia mecánica de la unión, siendo ésta más significativa en la unión con gap 50, motivado principalmente por la difusión del silicio y molibdeno del centro del cordón de soldadura al material base.

El método de calentamiento en horno de alto vacío se ha mostrado efectivo para la realización de uniones con excelentes propiedades mecánicas. En este sentido, hay que destacar que un vacío del orden de $1 \times 10^{-4}$ mbar es suficiente para la protección y soldeo de la aleación de níquel objeto de estudio. 


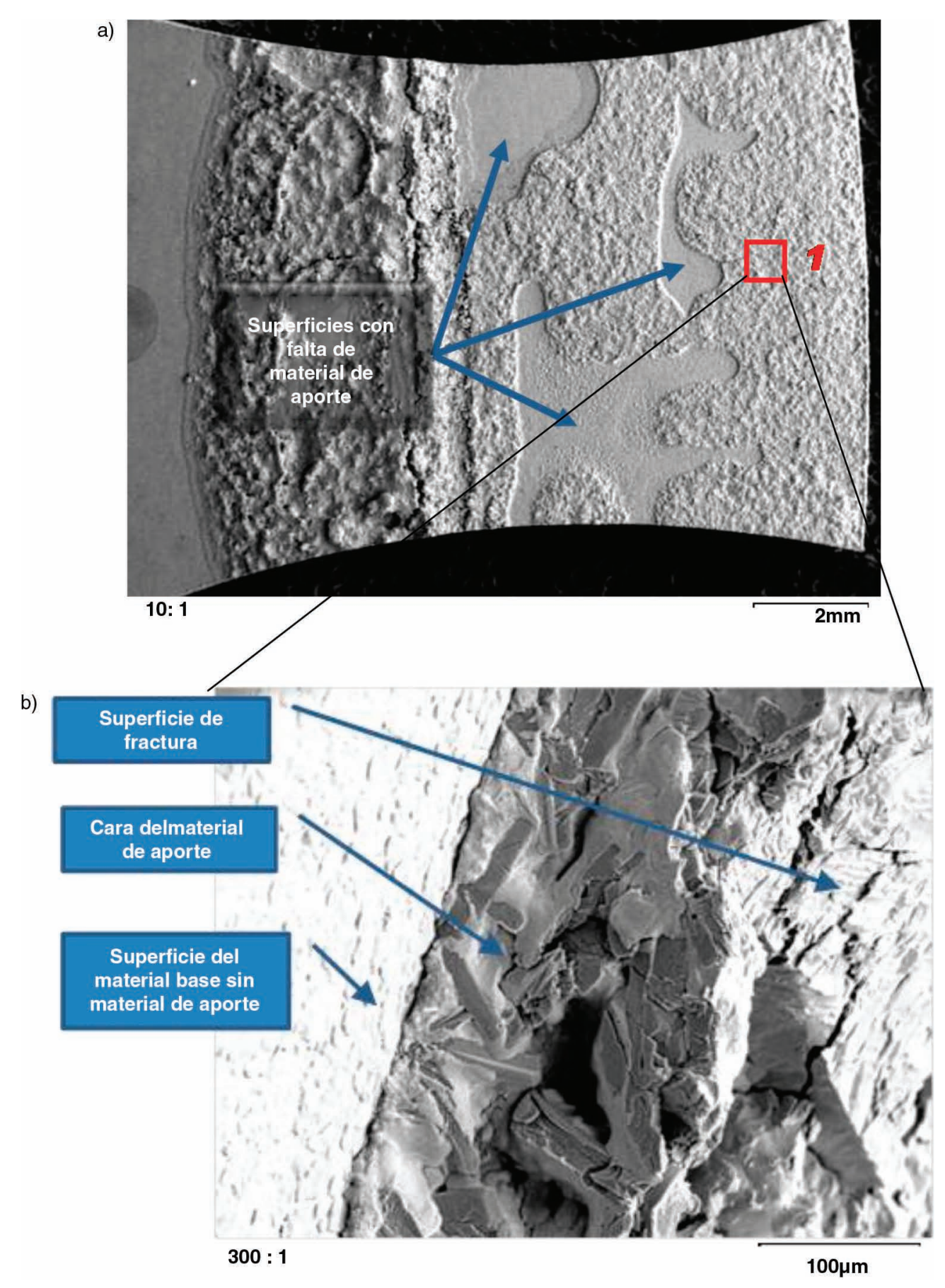

FigurA 5. a) Imagen SEM de la superficie de fractura de la unión con gap $200 \mathrm{y} \mathrm{t}_{\mathrm{Ts}} 10 \mathrm{y}$ b) Imagen SEM de la superficie de contacto aporte-material base.

TABLa 6. Resultados del ensayo de cizalla

\begin{tabular}{lcc}
\hline Gap & $\mathbf{t}_{\text {Ts }}(\mathbf{m i n})$ & $\begin{array}{c}\text { Tensión máxima } \\
(\mathbf{M P a})\end{array}$ \\
\hline 50 & 10 & 102,5 \\
& 90 & 137,1 \\
200 & 10 & 80,9 \\
& 90 & 92,7 \\
\hline
\end{tabular}

\section{REFERENCIAS}

Brooks, C.R., Wang, Y.M. (1989). Effect on the microstructure of aging Hastelloy B2 from 550 to $850^{\circ} \mathrm{C}$ for 1,200 hours. Metallogr. 23 (1), 57-86. http://doi.org./ $10.1016 / 0026-0800(89) 90040-2$.

Brooks, C.R., Wang, Y.M. (1990). Tensile properties and fractography of aged hastelloy $\mathrm{B} 2\left(550-850^{\circ} \mathrm{C}\right.$ for up to 1200 h). Mater. Charact. 25 (2), 185-197. http://doi.org./ 10.1016/1044-5803(90)90009-9. 
Cao, S., Brooks, C.R., Whittaker, G. (1994). The structure of the heat-affected zone in welds of a Ni-29 wt.\% Mo commercial alloy (Hastelloy B2). Mater. Charact. 33 (1), 21-32. http://doi.org./10.1016/1044-5803(94)90054-X.

Davis, J.R. (2000). Nickel, Cobalt, and their alloys. ASM Specialty Handbook, USA.

Grushko, B., Weiss, B. (1984). Structure of Vacuum Brazed BNi-5 Joint of Inconel 718. Metall. Trans. A. 15 (4), 609620. http://doi.org./10.1007/BF02644192.

Kim, Y.H., Kim, I.H., Kim, K.T., Sin, S.Y., Kwun, S.I. (2007). Identification of Phases in the Wide-Gap Region Brazed with BNi-3 Filler Metal Powder Using Electron Backscatter
Diffraction. Mater. Sci. Forum 544-545, 355-358. http:// doi.org./10.4028/www.scientific.net/MSF.544-545.355.

Rodríguez, G., García, I., Damborenea, J. (1998). Aleación superficial de superaleaciones base níquel mediante laser. Rev. Metal. 34 (2), 175-179. http://doi.org/10.3989/revmetalm.1998.v34.i2.684.

Roberts, P. (2003). Industrial Brazing Practice. CRC Press, UK. Schwartz, M. (2003). Brazing. $2^{\text {nd }}$ Edition. ASM International, USA

UNE-EN ISO 17672 (2010). Soldeo fuerte, metales de aportación. AENOR, España. 